\title{
ON THE STABILITY OF AN ALTERNATIVE FUNCTIONAL EQUATION
}

\author{
BOGDAN BATKO
}

Abstract. We deal with the stability of the alternative functional equation

$$
f(x+y)+f(x)+f(y) \neq 0 \Longrightarrow f(x+y)=f(x)+f(y) .
$$

Mathematics subject classification (2000): 39B82, 39B55.

Key words and phrases: Stability, conditional Cauchy equation, alternative equation.

\section{REFERENCES}

[1] B. BATKO, On the stability of Mikusiski's equation, Publ. Math. Debrecen, 66, (2005), 17-24.

2] B. BATKO, Stability of Dhombres' equation, Bull. Austral. Math. Soc., 70, (2004), 499-505.

[3] B. BATKO, JACEK TABOR, Stability of an alternative Cauchy equation on a restricted domain, Aequationes Math., 57, (1999), 221-232.

[4] B. BATKO, JACEK TABOR, Stability of the generalized alternative Cauchy equation, Abh. Math. Sem. Univ. Hamb., 69, (1999), 67-73.

[5] P. W. ChOlEwA, Stability as a property dependent upon an equation, not on a function in: Report of Meeting, The 34th International Symposium on Functional Equations, Aequationes Math., 53, (1997), 166.

[6] R. GER, On a characterization of strictly convex spaces, Atti Accad. Sci. Torino, Cl. Sci. Fis. Mat. Natur., 127, (1993), 131-138.

[7] R. GER, Functional equations with a restricted domain, Rend. Sem. Mat. Fis. Milano, 47, (1977), 175-184.

[8] D. H. HYERS, On the stability of the linear functional equation, Proc. Nat. Acad. Sci. U.S.A., 27, (1941), $222-224$

[9] D. H. HyERs, G. IsAC AND Th.M. Rassias, Stability of Functional Equations in Several Variables, Birkhäuser, Boston - Basel - Berlin, 1998.

[10] M. KuCZMA, Functional Equations on restricted domains, Aequationes Math., 18, (1978), 1-34.

[11] M. KuCZMA, On some alternative functional equations, Aequationes Math., 17, (1978), $182-198$.

[12] J. RÄTZ, On approximately additive mappings, General Inequalities 2 (Oberwolfach 1978), Birkhäuser, Basel - Boston, 1980, pp. 233-251.

[13] J. SCHWAIGER, Remark 13 in: Report of Meeting, The 41st International Symposium on Functional Equations, Aequationes Math., 67, (2004), 309.

[14] S. M. Ulam, A collection of mathematical problems, Interscience Publ., New York 1960. 